\title{
Assessment of medicine stock-outs challenges in public health services
}

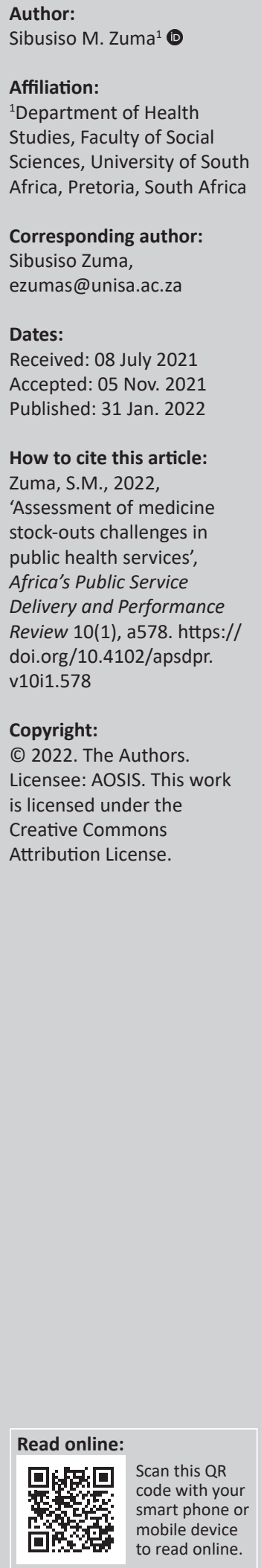

Background: Essential medicines are important in the provision of healthcare services. In order to promote effective management of diseases, essential medicines should be consistently available at various healthcare service points. However, in several parts of South Africa and other African countries, there are challenges with essential medicines provisioning, as evidenced by public health sector stock-outs occurring when patients visiting the healthcare services do not get all the medicine prescribed for their health conditions. This research investigated the problems involved in the provision of essential medicines in order to propose strategies that will reduce medicine stock-outs.

Aim: This study aimed to investigate the problems involved in the provision of essential medicines within the district healthcare services in order to propose strategies to promote reduction in medicine stock-outs.

Method: A qualitative exploratory research design was utilised. Data collection was carried out through semi-structured interviews with pharmaceutical managers identified as information-rich participants, as they have first-hand experience in the provision of essential medicines. The data were analysed using thematic analysis.

Results: The study identified the various aspects including inadequate appointment of postbasic pharmacist assistants and manual medicine stock management systems have a negative impact on medicines provisioning in the public health sector. Rural health facilities experience more challenges with essential medicines provisioning.

Conclusion: Based on the challenges identified, the study concludes that a comprehensive strategic framework should be implemented including placement of Post-Basic Pharmacist Assistants in the primary healthcare facilities and deployment of electronic medicine stock management system to facilitate consistent provisioning and availability of medicines in particular for the rural health facilities.

Keywords: health services; essential medicines; medicine supply management; primary health care; medicine stock-outs.

\section{Introduction}

Access to safe, effective and quality medicines and vaccines for all is one of the targets of the Sustainable Development Goals. Achieving universal health coverage requires access to safe, effective, quality and affordable essential medicines and vaccines. Access is a global concern in view of the increasing pressure on the ability of all health systems to provide full and affordable access to healthcare. Persisting problems of shortages and stock-outs of essential medicines, especially for noncommunicable diseases, and that of vaccines pose an unacceptable risk to public health (World Health Organization 2018).

Essential medicines provisioning consumes more than $60 \%$ of the total health budget in resourceconstrained settings. Availability of essential medicines to treat non communicable diseases (NCDs) was found to be below $60 \%$ in African region countries surveyed (World Health Organization 2016). No less than a third of the populations in the developing countries of Africa and Asia have limited access to essential medicines because of access challenges. These challenges have caused increasing medicine stock-outs and thus patients are not receiving a complete package of their prescribed medicines. When essential medicines are not provided, control of clinical conditions is compromised, which leads to incidents where healthcare service users experience medical complications and potential loss of life (World Health Organization 2014).

Medicins Sans Frontieres reported that in South Africa lack of access to medicines has complex underlying factors ranging from health programme implementation to medicine supply system challenges (Medicines Sans Frontiers 2013). 


\section{Background}

In South Africa the provision of essential medicines is a regulated function of the National Department of Health which is facilitated through national medicine contracts from which provinces and regions procure medicines for delivery to the central medical depots for distribution to the various healthcare facilities based on orders placed (South African Department of Health 2018).

The central medicine procurement and warehousing system has both benefits such as financial resource pooling and challenges such as long turnaround times for delivery. The challenges result in inconsistent provisioning and availability of essential medicines (Zuma 2017).

South Africa has experienced medicine provisioning challenges because there was a high prevalence of stock-outs across all nine provinces evidenced by the large interprovincial differences in stock-out occurrence, duration, and impact which suggest differences in the provincial ability to prevent, mitigate and cope with the medicine stock-outs (Hwang et al. 2019). The reasons cited for the medicine stock-outs were the following: delayed payment of medicine supplying companies, companies not meeting the order quantities placed by the government's medicine warehouses and poor procurement and logistics management skills (Stop Stock Outs Project 2016).

The National Department of Health Strategic Plan 2020-2025 regard essential medicines provisioning as an important government function to achieve communicable disease control. The government further identified medicine provisioning as a risk requiring mitigation through ringfenced budget and monitored medicine procurement contracts to promote the likelihood that essential medicines will always be available (South African Department of Health 2018).

In the 2012 National Health Facilities Baseline Audit, at least $86 \%$ of the primary healthcare facilities were reported to be without the full package of essential medicines for treating conditions such as HIV and AIDS, tuberculosis (TB) and chronic conditions, as well as lacking certain vaccines (South African Department of Health 2012). Another report published in December 2013 further highlighted the shortage of essential medicines across the country and cited the reasons as being the lack of a uniform, standardised framework for the provision of medicines in South Africa, especially for the district healthcare services under which primary healthcare facilities are located (SECTION 27 2013).

The 2011/12 and 2012/13 District Health Information System (DHIS) data, indicated that the stock-out rate for any TB medicine was estimated as $10.7 \%$ (ranging from $4.3 \%$ in the Western Cape to $25.4 \%$ in the Free State). By contrast, 2013 data from the National Stop Stock Outs Survey, a civil society conducted telephonic survey, showed a national stock-out rate of TB and HIV medicines of 21.5\% (Day et al. 2019).
Five provinces in South Africa, namely the Free State, Limpopo, Eastern Cape, Gauteng and Mpumalanga, experienced medicine shortages during the 2013/2014 and 2014/15 calendar years (Stop Stock Outs Project 2016). This finding was confirmed by the civic organisation report that revealed that medicine stock-outs, particularly in terms of the HIV or AIDS and TB medicines, were a national crisis affecting all nine provinces, with the rural provinces being the worst affected. In November 2013, the provinces had a stock-out rate of $54 \%$, as well as a $35 \%$ stock-out for vaccines (Medicines Sans Frontiers 2013).

\section{Methods \\ Problem statement}

Patients diagnosed with communicable and noncommunicable diseases require medicines to treat and maintain disease control. However, various provinces in South Africa experience problems with consistent provisioning of essential medicines. The resultant stock-outs, impact public health sector patients who do not receive their medication as prescribed. Medicines stock-out negatively affects the clinical management of diseases of patients.

\section{Research aim}

The researcher aimed to investigate the problems involved in the provision of essential medicines in order to propose strategies to promote reduction in medicine stock-outs.

\section{Research design and method}

A qualitative exploratory research design was utilised to conduct this study. Data collection was carried out through semi-structured interviews with pharmaceutical managers identified as information-rich participants, as they have first-hand experience in the provision of essential medicines. The data were analysed using an inductive model to categorise the data into similar themes and attach meaning to them.

\section{Research setting}

The study was conducted in eight of the nine provincial pharmaceutical units in South Africa; Limpopo province pharmaceutical unit declined participation despite the gatekeeper's permission granted for the study.

The pharmaceutical units were chosen because of their responsibility of coordinating the provision of essential medicines to the various healthcare facilities. Thus, these units were in a position to provide valuable input to the study.

\section{Population and sample}

The study population was the pharmaceutical managers who are responsible for the coordination of essential medicines provisioning in South Africa. The sample, comprising 15 managers made up of four females and 11 males was 
purposively selected to obtain rich data about the study topic. They included eight provincial heads of pharmaceutical services and seven medical depot managers. The managers had more than five years' experience in the management of medicine provisioning.

\section{Data collection}

Data collection was carried out through individual interviews in eight provinces. The interviews were conducted in the various provincial pharmaceutical offices and medicine warehouses. The interviews were tape-recorded by the researcher.

The data collection tool was a semi-structured interview guide developed based on the medicine supply framework covering the following aspects: selection, procurement, distribution, rational medicine use and policy framework. The interview guide was used to record responses to key discussion points, as well as to record observations of the activities involved in the provision of essential medicines.

The physical interviews were chosen to allow for exploration of the full range of communication aspects, including responses to non-verbal signs observed (Harding 2013). The researcher reviewed the data collected and reflected on the practices observed and audio-recordings made during the site visits.

\section{Data analysis}

The audio data were then professionally transcribed to produce a transcription record of the digital recordings and notes (Marshall \& Rossman 2011). The data were then organised and analysed by means of thematic analysis resulting in themes and meaningful categories for the study.

The stages followed during the qualitative data analysis process included reading, rereading the data and comparing aspects to understand the content, taking into account the research aim and questions underlying key segments providing descriptive comments matching the identified segments across the database, attaching labels and identifying sub-groups and finally assessing the grouping against the current literature and theory with a view to presenting the research findings (Grbich 2013).

\section{Ethical considerations}

Ethical clearance for this study was obtained from University of South Africa Health Research Ethics Committee reference HSDC/370/2014 and gatekeepers' permission was obtained from the nine research committees of the various provinces. The elements of trustworthiness, credibility, confirmability, authenticity and transferability were ensured in conducting the study. All participants provided informed consent for participation in the study.

\section{Results and discussion Demographic profile of the participants}

Fifteen managers, four females and 11 males, participated in the study. Eight were the Heads of Pharmaceutical Services whilst seven were Medical Depot Managers. The average experience in the medicine provisioning management was more than five years for each of the participants.

\section{Themes}

The following five themes emerged from the study:

1. Rural health facilities experience more essential medicine provisioning challenges

2. Poor medicine stock control result in stock losses

3. Suboptimal manual medicine stock management systems

4. Absence of permanent post-basic pharmacist assistants negatively affects the provision of essential medicines in primary health clinics

5. Best practices observed

The above themes will be discussed in the following subsections.

\section{Rural health facilities experience more essential medicines provisioning challenges}

The participants reported that owing to rural areas' having poor road infrastructure and an inability to attract and retain pharmaceutical service personnel, the provision of essential medicines is inconsistent in the rural primary health clinics when compared with those in the urban areas.

\section{One rural province participant stated the following: \\ 'I am alone for all those things and the depot's also working with minimal staff as we are all experiencing staff shortage but we are expected to provide all the services that are requested.' (Participant 3, head of pharmaceutical services, female)}

This challenge is confirmed by Modisakeng et al. (2020) who states that although there have been some successes over the past three years and several initiatives undertaken by the government to improve the supply chain for medicines, stock-outs at primary healthcare facilities remain an undeniable threat to the health of the people of South Africa.

Those most vulnerable to the effects of stock-outs are usually poor and rural communities who depend on public facilities for healthcare services. When the rural facilities experience stock-outs, the impact goes beyond health. Patients often make repeated, costly trips to health facilities to keep up their prescriptions; pharmacists and nurses spend more time rationing drugs instead of caring for ill patients; and babies are sometimes not vaccinated (Stop Stock Outs Project 2016).

\section{Poor medicine stock control result in stock losses}

Some participants confirmed that there is poor medicine stock control as they observe when conducting health facilities 
visit that some medicine rooms are freely accessible resulting in opportunities for medicine theft, inconsistent stock replenishment and periodic stock-outs in particular for the facilities with no pharmacy personnel dedicated to medicine stock management and control. All of these problems were considered to be a result of poor management.

One participant suggested that the issue of poor management can be resolved through the deployment of dedicated personnel in the form of Pharmacists and Pharmacist Assistants:

'Pharmacist Assistant should be deployment to primary health care (PHC) level and Pharmacist in the hospitals to control medicine orders.' (Participant 4, head of pharmaceutical services, male)

Zuma (2013) reported that, the supply of medicines within the public sector is plagued by theft and a lack of control, as evidenced by medicines intended to be used exclusively in public health facilities being found in the private sector.

Matsoso, Fryatt and Andrews (2015) further advocate that District Pharmacists should be appointed in all districts, as various studies suggest that poor medicine control and stockouts occur at facility level without Pharmaceutical Services oversight. The aforementioned authors also believe that district pharmacists could play a more influential role in medicine provisioning, provided that they are adequately resourced and recognised as key partners in district healthcare services.

The researcher identified that in the Eastern Cape and Northern Cape there were districts without an appointed District Pharmacists and also the majority of the primary healthcare facilities were without at least one Pharmacist Assistant.

\section{Suboptimal manual medicine stock management systems}

The participants reported that the primary healthcare facilities mostly use manual stock management systems. Medicine rooms do not always comply with Good Pharmacy Practice - in terms of space and temperature control - for effective medicine stock management. This results in the ordering of less-than-required quantities to meet the demand for at least one month. The suboptimal storage space also has an impact on stock-taking and rotation to minimise the expiry of medicines and the identification of impending stock-outs.

Two participants commented as follows:

'We have challenges in the PHC level due to limited infrastructure and IT systems. If there is a shortage of a particular item, it takes forever to resolve of that particular item.' (Participant 1 , medical depot manager, male)

'Order replenishment and good stock management is dependent on the IT system with minimum and maximum stock levels instead of awaiting orders from the health facilities enabling both stock control and relieve facility staff for order placement and follow up time.' (Participant 3, head of pharmaceutical services, female)
A study conducted on the pharmaceutical management of TB in seven of the nine provinces found that the provision of essential medicines was suboptimal. The availability of ARV and $\mathrm{TB}$ medication was at less than $95 \%$ in five of the provinces, namely the Free State, Eastern Cape, Gauteng, Limpopo and Mpumalanga. The factors contributing to this low availability, especially in nurse-led primary health clinics, include poor stock control, poor management and a lack of electronic stock management systems, as manual stock cards were found to be out of date at most facilities (Pure Health Consulting 2012).

Another study conducted in the Free State province demonstrated that there is inadequate availability of medicine in district healthcare services owing to factors such as a lack of delegation and all the red tape involved in the procurement of medicines, as well as the lack of an electronic medicine procurement and monitoring system. It has been proposed that innovative plans and models be developed to promote consistent medicine availability, especially for the District Health System through which the majority of citizens access healthcare services in primary health clinics. The following factors negatively affect the availability of medicines: poor medicine stock management, late deliveries from the medical depot, poor communication between the role-players, a lack of electronic ordering systems, duplication of patients, medicine theft, transport challenges and departmental red tape (Zuma 2013).

Kheirandish et al. (2015) state that ensuring a reliable healthcare and supply system for the provision of medicines is critical and can be realised through monitoring enhancement to identify more rapidly and accurately the medicine needs and shortages. These approaches include monthly collections of data regarding medicine consumption or distribution; establishing an immediate warning system to detect shortages; expanding the data collection network to all parts of the country and establishing an information centre for medicine shortages.

\section{Absence of permanent post-basic pharmacist assistants negatively affects the provision of essential medicines in primary health clinics}

All the participants agreed that primary health clinics without dedicated permanently appointed pharmacist assistants to manage medicine provisioning are bound to experience challenges and medicine stock losses, as nurses focus more on clinical care than on medicine stock management.

The participants commented as following:

'Health facilities have Pharmacy related posts which are inadequately filled to provide medicine management support and guidance.' (Participant 10, head of pharmaceutical services, male)

'[The] appointment of dedicated pharmacist assistants for PHC facilities is key if the stock outs are to be minimised.' (Participant 2 , head of pharmaceutical services, male) 
For efficient medicine provision, the South African Pharmacy Council advocates the availability of pharmacists and pharmacist assistants to take charge of the provision of medicines in hospitals, community health centres and primary health clinics in an environment that complies with Good Pharmacy Practice (South African Pharmacy Council 2020). However, in practice there have been several challenges regarding the availability of pharmacists and pharmacist assistants in public health facilities, with many hospitals and community health centres not having permanent pharmacists. In primary health clinics, nurses are usually the only available personnel responsible for the provision, ordering, control and enabling access to medicines (International Council of Nurses 2011).

\section{Best practices observed}

During the site visits across the provinces, at least two provinces, namely the Western Cape and North West reported more consistent provisioning and availability of essential medicines. The factors that contributed to this best practice included electronic stock management; maintenance of a 3-month buffer stock; close monitoring of and weekly reporting on stock availability; active contract management and interaction with the suppliers over quantification, shortages and forecasting of needs; involvement of all role-players in medicine stock management, including finance managers, health programme managers and pharmacists; as well as the adjustment of issuing quantities during limited stock periods to ensure that all patients receive medication while waiting for the stock replenishment.

The findings were consistent with a countrywide study conducted by Pure Health Consulting in 2012.

Some participants indicated the following:

'We have Stock Management group and transport to shift stock around.' (Participant 8 , head of pharmaceutical services, female)
'I would say something that stands out from our province is having our own finance unit because I hear a lot of provinces complaining that they send the payments to provincial office, to the Chief Financial Officer (CFO's) office, and it's not prioritised.' (Participant 9 , medical depot manager, male)

\section{Proposed strategies to address the challenges identified}

To address the challenges identified and promote access to essential medicines that are vital for healthy living for all, the researcher recommends that the strategies summarised in Table 1 be implemented to guide the provisioning of essential medicines within public healthcare services.

\section{Strengths and limitations of the study}

The strength of the study is that the study was conducted at a provincial level with the decision makers in medicine provisioning in the form of Pharmaceutical Services Managers.

The limitation is that the experiences of the operational managers were not obtained which could have provided frontline data on the medicine provisioning problems in the public health sector especially the rural areas which are mostly hit by medicine shortages.

\section{Study implications for clinicians and policy makers}

The study provides clinicians and policymakers with a report on the challenges encountered in the provisioning of essential medicines as well as provides policymakers a proposed framework to promote better provisioning of essential medicines.

TABLE 1: Proposed strategies to improve the provisioning of essential medicines.

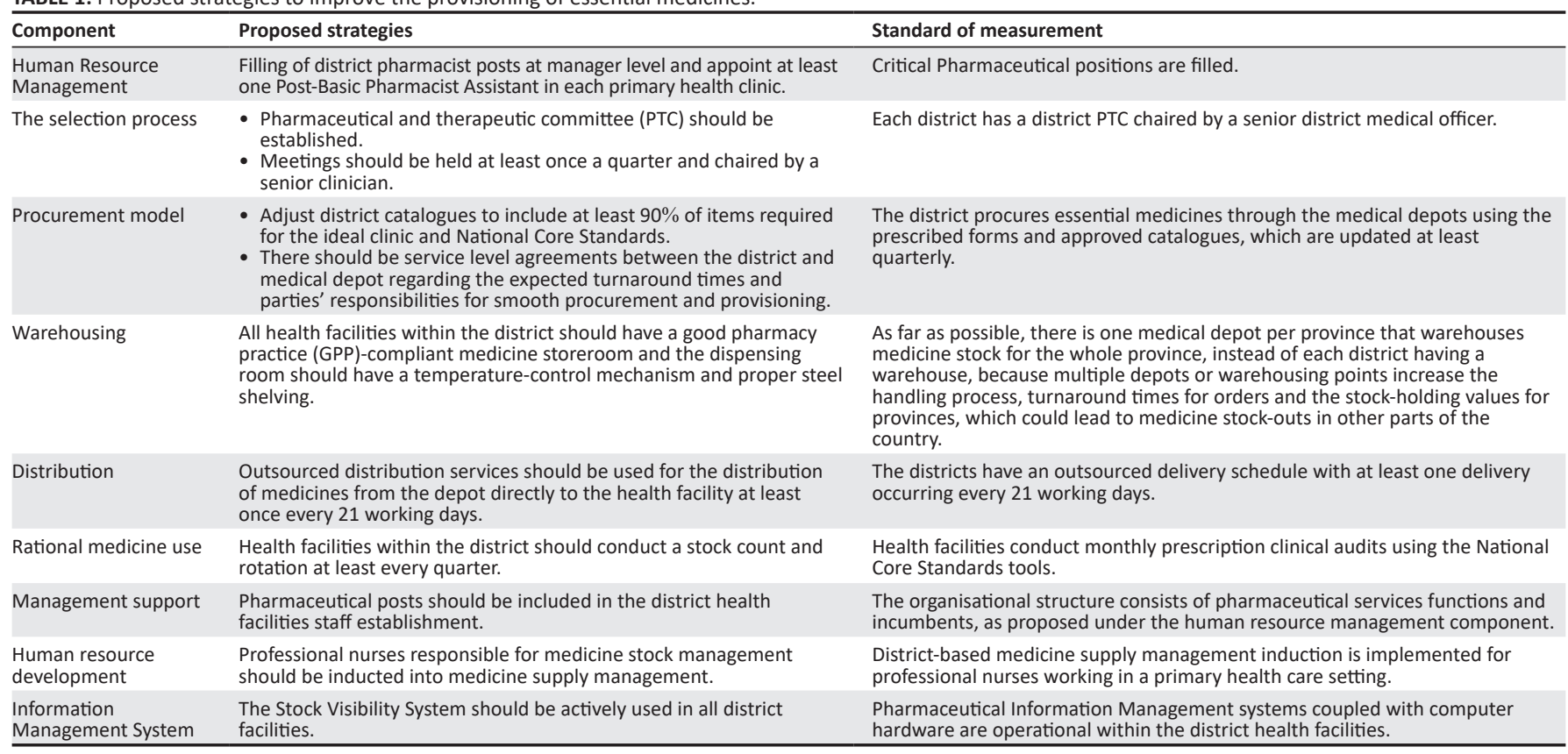

Source: Zuma, S.M., 2017, 'Framework for provision of essential medicines within district health service province', Unpublished doctoral thesis, University of South Africa, Pretoria. 
The researcher believes that the findings and proposed strategies will go a long way towards ensuring that service users have improved access to essential medicines in order to control their respective health conditions and diseases. This, in turn, should increase the life expectancy of the service users.

\section{Conclusion}

Based on the experiences of the study participants, it is evident that the South African medicine provisioning challenges are not exclusively related to medicine budget constraints but occur as a result of inadequate systems to manage the medicine stock in the chain from the medicine warehouses to the public health facilities. The inadequate systems include human resources in the form of Pharmacist Assistants as well as the use of manual medicine stock management system within public health sector.

Based on the challenges identified, the study concludes that a comprehensive strategic framework should be implemented including placement of Post-Basic Pharmacist Assistants in the primary healthcare facilities and deployment of electronic medicine stock management system that will contribute towards promoting consistent provisioning and availability of medicines in particular for the rural health facilities.

Further research can be conducted about the experiences of the managers of public health facilities on the challenges related to medicine provisioning as well as their suggestions to promote consistent supply of medicines which are essential in the treatment and management of prevailing diseases.

\section{Acknowledgements Competing interests}

The author declares that he has no financial or personal relationships that may have influenced him inappropriately in writing this article.

\section{Author's contributions}

S.M.Z. the sole author of this research article.

\section{Funding information}

This research received no specific grant from any funding agency in the public, commercial or not-for-profit sectors.

\section{Data availability}

Data sharing is not applicable to this article as no new data were created or analysed in this study.

\section{Disclaimer}

The views and opinions expressed in this article are those of the author and do not necessarily reflect the official policy or position of any affiliated agency of the author.

\section{References}

Day, C., Gray, A., Ndlovu, N. \& Cois, A., 2019, Health and related indicators 2019: Interrogating the UHC service coverage index, Health System Trust, Durban.

Grbich, C., 2013, Qualitative data analysis: An introduction, 2nd edn., Sage, London.

Harding, J., 2013, Qualitative data analysis: From start to finish, Sage, Los Angeles, CA.

Hwang, B., Shroufi, A., Gils, T., Steele, S.J., Grimsrud, A. \& Boulle, A. et al., 2019, 'Stockouts of antiretroviral and tuberculosis medicines in South Africa: A national crosssectional survey', PLoS One 14(3), e0212405. https://doi.org/10.1371/journal. pone.0212405

International Council of Nurses, 2011, Closing the gap: Increasing access and equity, ICN, Geneva.

Kheirandish, M., Rashidian, A., Kebriaeezade, A., Majid, A., Cheraghali, M.A. \& Soleymani, F., 2015, 'Review of pharmaceutical policies in response to economic Soleymani, F., 2015, 'Review of pharmaceutical policies in response to economic
crises and sanctions', Iran: Journal of Research in Pharmacy Practice 4(3), 115-122. https://doi.org/10.4103/2279-042X.162361

Marshall, C. \& Rossman, G.B., 2011, Designing qualitative research, 5th edn., Sage, CA.

Matsoso, M.P., Fryatt, R.J. \& Andrews, G., 2015, The South African health reforms, 2009-2014: Moving towards universal coverage, Juta, Cape Town.

Medicines Sans Frontiers, 2013, Treatment action campaign, Section 27 and rural health advocacy project. Emergency intervention at Mthatha depot: The hidden cost of inaction, Eastern Cape Republic of South Africa: MSF, Mthatha.

Modisakeng, C., Matlala, M., Godman, B. \& Meyer, J.C., 2020, 'Medicine shortages and challenges with the procurement process among public sector hospitals in South Africa; findings and implications', Biomedical Central Health Services Research 20, 234. https://doi.org/10.1186/s12913-020-05080-1

Pure Health Consulting, 2012, Pharmaceutical management of TB in South Africa; rapid assessment conducted in 7 provinces, Systems for Improved Access to Pharmaceuticals and Services (SIAPS) Program, Management Sciences for Health, Arlington, VA.

South African Department of Health, 2012, National health facilities baseline audit report, Government Printers, Pretoria.

South African Department of Health, 2018, Strategic plan 2020-2025, Government Printers, Pretoria.

South African Pharmacy Council, 2020, Good pharmacy practice in South Africa, 4th edn., SAPC, Arcadia, CA.

SECTION 27, 2013, Monitoring our health: An analysis of the breakdown of healthcare services in selected Gauteng facilities, SECTION 27, Johannesburg.

Stop Stock Outs Project, 2016, 2015 stock outs national survey. Third annual report South Africa, Stop Stock Out, Johannesburg.

World Health Organization, 2014, Essential medicines and health products biennial report 2012-2013, WHO, Geneva.

World Health Organization, 2016, Annual report 2016: WHO essential medicines and health products, WHO, Geneva.

World Health Organization, 2018, Addressing the global shortage of and access to medicines and vaccines, WHO, Geneva.

Zuma, S.M., 2013, 'Factors contributing to non-availability of essential medicines within the Free State district health service province', Unpublished master's dissertation, University of South Africa.

Zuma, S.M., 2017, 'Framework for provision of essential medicines within district health service province', Unpublished doctoral thesis, University of South Africa. 